\title{
Anterior capsule contraction and intraocular lens decentration and tilt after hydrogel lens implantation
}

\author{
Ken Hayashi, Hideyuki Hayashi, Fuminori Nakao, Fumihiko Hayashi
}

\begin{abstract}
Aim-To prospectively investigate changes in the area of the anterior capsule opening, and intraocular lens (IOL) decentration and tilt after implantation of a hydrogel IOL.

Methods-100 patients underwent implantation of a hydrogel IOL in one eye and an acrylic IOL implantation in the opposite eye. The area of the anterior capsule opening, and the degree of IOL decentration and tilt were measured using the Scheimpflug videophotography system at 3 days, and at 1, 3, and 6 months postoperatively.

Results-The mean anterior capsule opening area decreased significantly in both groups. At 6 months postoperatively, the area in the hydrogel group was significantly smaller than that in the acrylic group. The mean percentage of the area reduction in the hydrogel group was also significantly greater than that in the acrylic group, being $16.9 \%$ in the hydrogel group and $8.8 \%$ in the acrylic group. In contrast, IOL decentration and tilt did not progress in either group. No significant differences were found in the degree of IOL decentration and tilt throughout the follow up period.

Conclusions-Contraction of the anterior capsule opening was more extensive with the hydrogel IOL than with the acrylic IOL, but the degree of IOL decentration and tilt were similar for the two types of lenses studied.
\end{abstract}

(Br F Ophthalmol 2001;85:1294-1297)

Hayashi Eye Hospital, Fukuoka, Japan

K Hayashi

F Nakao

F Hayashi

Department of

Ophthalmology,

School of Medicine,

Fukuoka University,

Fukuoka, Japan

H Hayashi

Correspondence to:

Ken Hayashi, Hayashi Eye Hospital, 4-7-13

Hakataekimae, Hakata-Ku, Fukuoka 812, Japan

hayashi-ken@hayashi.or.jp

Accepted for publication 29 May 2001
When performing small incision cataract surgery, ophthalmic surgeons are now able to choose many types of foldable intraocular lenses (IOLs). At present, the optics of most foldable IOLs are made of silicone or soft acrylic. Recently, a foldable IOL with the optic composed of a hydrogel copolymer (Hydroview, H60M, Bausch \& Lomb, St Louis, MO, USA) has become commercially available. It has been shown that hydrogel lenses have excellent biocompatibility since they are hydrophilic, ${ }^{12}$ and few inflammatory cells are deposited on their surface. ${ }^{3}{ }^{4}$ However, because of one piece design or an weak adhesive quality of hydrogel materials to the lens capsule, ${ }^{5}$ one type of plate haptic hydrogel IOL (Iogel; Alcon Laboratories, Fort Worth, TX, USA) made of poly(2-hydroxyethyl methacrylate) (HEMA), of which $38 \%$ was water, tended to become decentred. ${ }^{6-9}$ The Hydroview IOL has a three piece open loop design with a hydrogel optic composed of 2-hydroxyethyl methacrylate and 6-hydroxyhexyl methacrylate, and has a water content of $18 \%$. The Hydroview IOL, made of a different hydrogel material and with a different design, may preclude or avoid the problems encountered with the earlier hydrogel IOL.

It has been reported that postoperative changes of the lens capsule, including anterior capsule contraction and fibrosis ${ }^{10}{ }^{11}$ and posterior capsule opacification ${ }^{12-15}$ vary substantially depending upon the optic material of the implanted lenses. For example, we have shown that anterior capsule contraction is more extensive with a silicone IOL than with a poly(methylmethacrylate) or acrylic IOL. ${ }^{10}$ An extensive contraction of the anterior capsule opening certainly restricts the examination of the peripheral retina and can also impair the visual function. Furthermore, previous studies demonstrated the hydrogel IOL to be associated with more opacification of the posterior capsule. ${ }^{16}{ }^{17}$ Thus, a better understanding of the interaction between the optic material and the lens capsule is essential for appropriate selection of an IOL.

The objective of the study was to prospectively examine reduction of the anterior capsule opening in the presence of a hydrogel IOL, and the degree of IOL decentration and tilt. In this study, the degree of these changes were compared with those of soft acrylic IOLs, which served as controls and which were implanted in the same manner in the opposite eye.

Patients and methods

All patients who were consecutively admitted to the Hayashi Eye Hospital for bilateral cataract surgery between December 1999 and June 2000 were screened for inclusion in this study. Preoperative exclusion criteria were: (1) cataracts due to causes other than age, (2) complicated cases scheduled for extracapsular or intracapsular cataract extraction, (3) a history of ocular surgery or inflammation, (4) ocular pathologies such as diabetic retinopathy and retinitis pigmentosa, (5) pseudoexfoliation syndrome, and (6) a pupil diameter measuring less than $6.0 \mathrm{~mm}$; (7) patients who could not be available for follow up were also excluded. Patient screening was continued until 100 patients who were to undergo phacoemulsification and IOL implantation were recruited.

The study protocol was approved by the institutional review board, and written informed consent was obtained from each 
Table 1. Mean (SD) area of the anterior capsule opening $\left(\mathrm{mm}^{2}\right)$ in the hydrogel and acrylic IOL groups

\begin{tabular}{llll}
\hline & Hydrogel group & Acrylic group & p Value \\
\hline 1 week & $26.9(3.7)$ & $26.3(3.5)$ & $0.2533^{\star}$ \\
1 month & $24.5(5.0)$ & $24.3(5.0)$ & $0.7067^{\star}$ \\
3 months & $22.4(5.3)$ & $23.4(4.9)$ & $0.1979^{\star}$ \\
6 months & $22.6(5.3)$ & $24.1(4.7)$ & $0.0444^{\dagger}$ \\
p Value & $<0.0001 \ddagger$ & $<0.0001 \ddagger$ & \\
\hline
\end{tabular}

^No statitical significance.

†Statitically significant difference between the groups.

¥Statistically significant difference between the postoperative points.

patient. All patients were allocated the day before surgery, by random number tables, to one of two groups: (A) those who received a hydrogel IOL (Hydroview, H60M) in the left eye and a hydrophobic acrylic IOL (AcrySof, MA60BM, Alcon Surgical Inc, Fort Worth, TX, USA) in the right eye, and (B) those who were implanted with a hydrogel IOL in the right eye and with an acrylic IOL in the left eye. Patients, examiners, and the surgeon were masked to randomisation. The H60M has a round $6.0 \mathrm{~mm}$ hydrogel optic and poly(methylmethacrylate) haptics; the MA60BM has a round $6.0 \mathrm{~mm}$ hydrophobic acrylic optic and poly(methylmethacrylate) haptics.

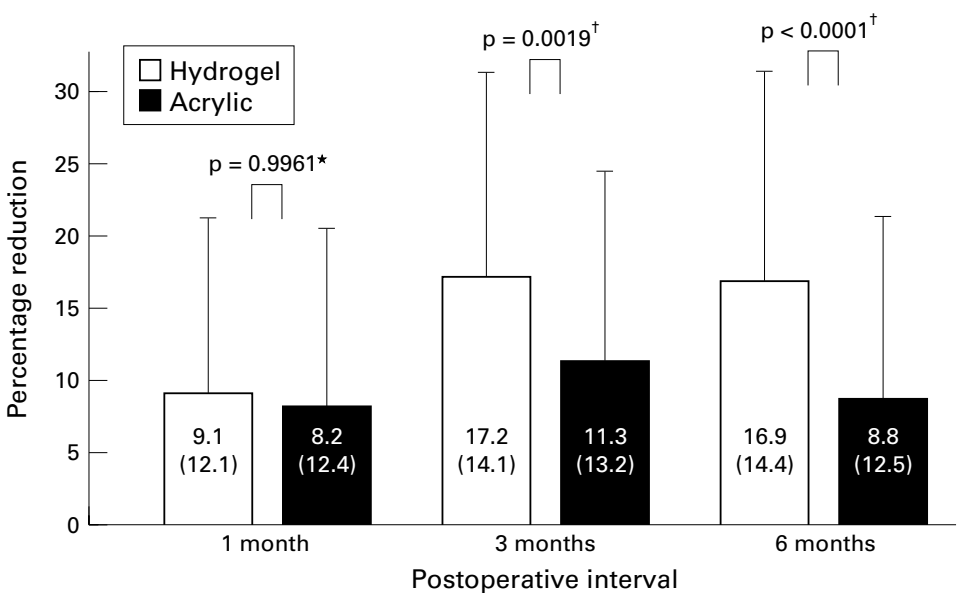

Figure 1 Comparison of the mean percentage of the area reduction between the hydrogel and acrylic IOL groups. The mean percentage of the area reduction in the hydrogel group was significantly greater than that in the acrylic group at 3 and 6 months after surgery.

*Not statistically significant, + statistically significant.

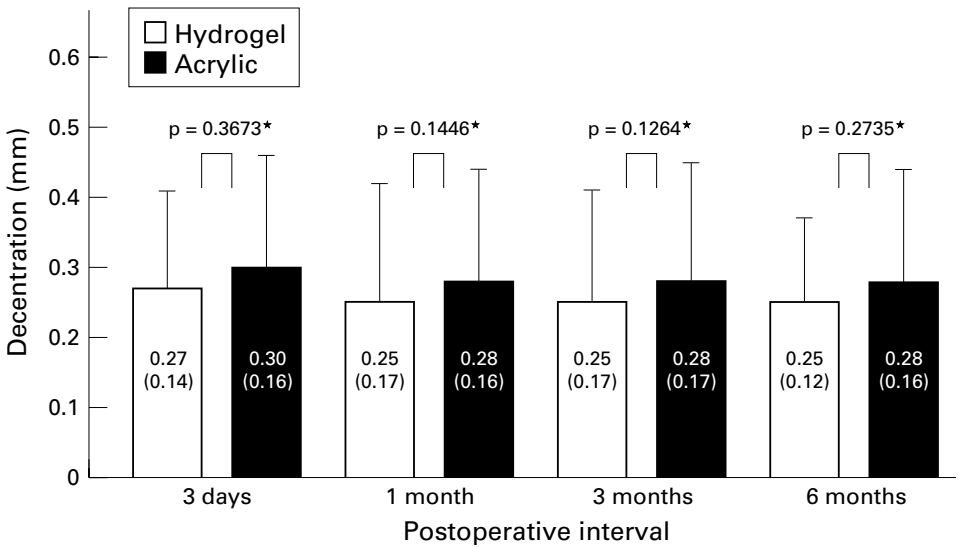

Figure 2 Comparison of the mean length of decentration ( $\mathrm{mm}$ ) between the hydrogel and acrylic groups. Mean decentration length did not progress either in the hydrogel group ( $p=$ 0.8076 , repeated measures ANOVA) or in the acrylic group $(p=0.9475)$. No statistically significant difference was observed in decentration between the two groups. ${ }^{\star} N o t$ statistically significant.
All surgeries were performed by the same surgeon $(\mathrm{KH})$ using standardised surgical procedures and medications. An approximately $3.5 \mathrm{~mm}$ scleral pocket incision was made for implantation of either IOL. After the incision, a $5.5 \mathrm{~mm}$ continuous capsulorhexis was created using a 25 gauge bent needle with $1 \%$ sodium hyaluronate. Following a thorough hydrodissection, the nucleus was emulsified and the cortical material aspirated completely. The lens capsule was reformed with sodium hyaluronate and the IOL placed into the capsular bag. In no case were any sutures placed. Eyes with surgical complications, including anterior capsule tears, posterior capsule rupture, and zonular dialysis, were excluded from the analysis.

Patients underwent examinations to measure the anterior capsule opening, and the degree of IOL decentration and tilt at 3 days, and at 1,3 , and 6 months after surgery using the Scheimpflug videophotography system (EAS-1000; Nidek Inc, Gamagori, Japan). The methods to measure the anterior capsule opening area, as well as the decentration length and tilt angle using the EAS-1000 system have all been described in previous studies. ${ }^{10}{ }^{18}$ All measurements with the EAS- 1000 system were carried out by five experienced ophthalmic technicians who were unaware of the purpose of this study.

Statistical analyses were performed to compare differences in the measurement data between the hydrogel and acrylic IOL groups using the Mann-Whitney U test. The repeated measures analysis of variance (ANOVA) was also used to compare differences between the various time points. Discrete parameters were compared using the $\chi^{2}$ test. Any differences showing a $p$ value of less than 0.05 were considered to be statistically significant.

\section{Results}

Of the 100 enrolled patients, 93 completed the 6 month follow up; seven patients did not undergo examination at 6 months after surgery. The average patient age was 71.4 (SD 6.5 ) years old, with a range of $52-85$ years. There were 34 men and 66 women. No statistically significant difference was found regarding age between the hydrogel and acrylic IOL groups. At the first postoperative examination, after full mydriasis at 3 days after surgery, all IOLs were confirmed to be implanted in the capsular bag.

Table 1 summarises the mean area of the anterior capsule opening in the hydrogel and acrylic groups. The mean opening area decreased significantly after implant surgery in both groups ( $\mathrm{p}<0.0001)$. The Mann-Whitney $\mathrm{U}$ test showed no significant difference between groups in the mean area at 3 days, or at 1 and 3 months after surgery. However, at 6 months the opening area in the hydrogel group was significantly smaller than that in the acrylic group $(p=0.0444)$. Furthermore, the mean percentage of area reduction in the hydrogel group at both 3 and 6 months after surgery was significantly greater than that in the acrylic group (Fig 1). 


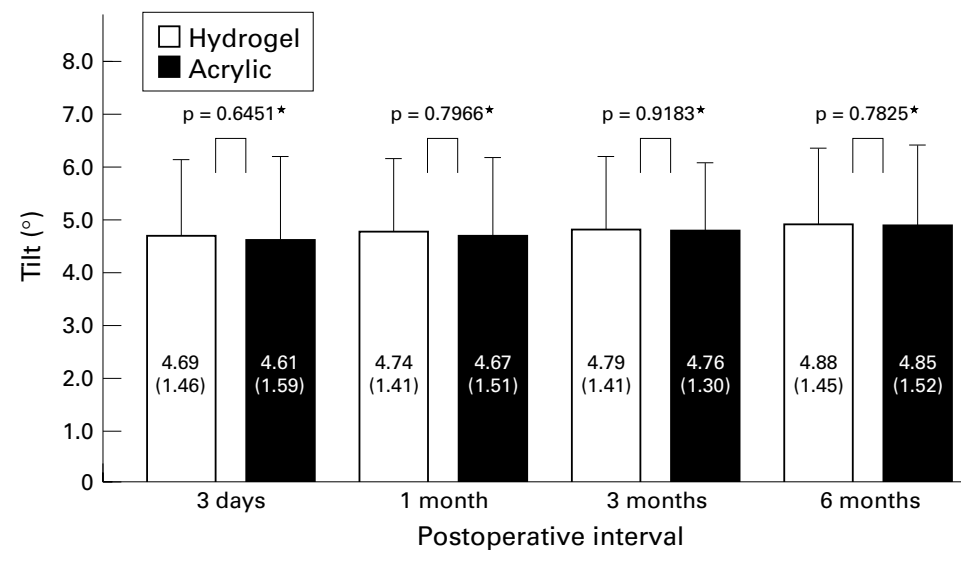

Figure 3 Comparison of the mean angle of tilt $\left({ }^{\circ}\right)$ between the hydrogel and acrylic IOL groups. Mean angle of tilt did not change in either group. No significant difference was observed between the hydrogel and acrylic groups. ${ }^{\star}$ Not statistically significant.

Figure 2 displays the mean length of IOL decentration in the two groups. The mean length of decentration did not progress in either group $(p=0.8076$ in the hydrogel group and $p=0.9475$ in the acrylic group, repeated measures ANOVA). Furthermore, no statistically significant difference was found in decentration between the hydrogel and acrylic groups throughout the 6 month follow up period.

Figure 3 shows the mean degree of IOL tilt. The mean angle of tilt did not progress in either group $(\mathrm{p}=0.6754$ in the hydrogel group and $p=0.2819$ in the acrylic group), and there was no significant difference in tilt angle between the two groups.

Four eyes $(4.0 \%)$ in the hydrogel group underwent neodymium $(\mathrm{Nd})$ :YAG laser anterior capsulotomy within the 6 month follow up, while only one eye $(1.0 \%)$ required laser treatment in the acrylic group. The mean opening area of the eyes that underwent anterior capsulotomy was 11.3 (SD 5.9) $\mathrm{mm}^{2}$, and the average interval between implant surgery and anterior capsulotomy was 4.4 (2.3) months. There was no significant difference in the survival curve of eyes not requiring anterior capsulotomy between the two groups $(\mathrm{p}=$ 0.1733, Mantel-Cox log rank test).

Figure 4 illustrates representative retroillumination photographs at 6 months of eyes in the hydrogel and acrylic groups, respectively. In the eye with a hydrogel IOL, fibrosis of the anterior capsule opening margin is marked (Fig 4A), whereas in the opposite eye, with the acrylic IOL, fibrosis of the anterior capsule is much less (Fig 4B).

\section{Discussion}

Hydrogel materials are reported to be highly biocompatible in terms of their hydrophilic properties. ${ }^{1}{ }^{2}$ However, a former hydrogel IOL with one piece plate haptic design had a high incidence of marked decentration. ${ }^{6-9}$ Furthermore, the serious complication of posterior luxation into the vitreous cavity following an $\mathrm{Nd}$ :YAG capsulotomy was reported. ${ }^{19}$

The Hydroview IOL, made of a hydrogel material different from that of the former IOL
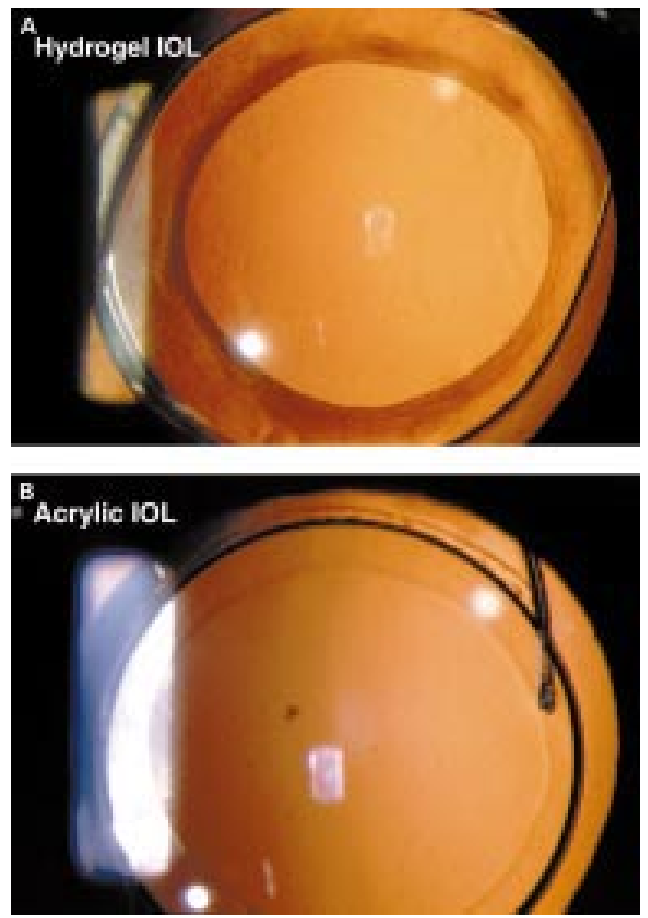

Figure 4 Retroillumination photographs showing bilateral eyes of a typical patient at 6 months after surgery. (A) In an eye with a hydrogel IOL, fibrosis of the anterior capsule, particularly along the capsulorhexis margin, is extensive. (B) In an opposite eye with an acrylic IOL, the anterior capsule fibrosis is less than that with the hydrogel IOL.

and with an open loop design, is now commercially available. It has been reported that this IOL has a unique clinical feature that results in extensive proliferation of lens epithelial cells on the surface, ${ }^{40-22}$ suggesting that this IOL has distinct surface properties. In this prospective study, we examined the extent of anterior capsule contraction and of IOL decentration and tilt due to capsular shrinkage.

Our study demonstrated that reduction of the anterior capsule opening area with the hydrogel IOL was greater than that with the acrylic IOL. The percentage of the area reduction at 6 months after surgery in the hydrogel group was approximately $17 \%$, which was almost double that of the acrylic group. With reference to our previous report, ${ }^{10}$ the percentage of reduction with the hydrogel IOL was similar to that with the silicone IOL, but greater than that with the poly(methylmethacrylate) IOL. Furthermore, fibrosis of the anterior capsule, particularly along the opening margin, was more pronounced with the hydrogel IOL than with the acrylic IOL. More importantly, $4.0 \%$ of eyes with a hydrogel IOL required Nd:YAG laser anterior capsulotomy, while only $1.0 \%$ of eyes with the acrylic IOL required this additional procedure. These results indicate that the hydrogel IOL has certain disadvantages in terms of anterior capsule contraction.

In contrast, neither the hydrogel nor acrylic IOLs showed progression of decentration and tilt. Furthermore, the degree of IOL decentration and tilt of the two lenses was almost the same. Menapace et $a l^{23}$ reported that a certain model of the former hydrogel IOL was 
not so markedly decentred in the capsular bag. Our results confirm that the Hydroview IOL with open loop design showed excellent centration so long as the IOL was placed in the bag.

The biological characteristics of the hydrogel IOL include high biocompatibility but weak adhesion to the lens capsule. As to the biocompatibility, many studies reported an extensive and persistent outgrowth of lens epithelial cells onto the IOL surface. ${ }^{40-22}$ In this series, we found only a slight membranous growth of lens epithelial cells which was not clinically relevant, although we cannot explain this. As to weak adhesion with the capsule, this can allow the space for synthesis of extracellular matrix due to myofibroblast ${ }^{25-27}$ and, therefore, may lead to dense fibrosis under the anterior capsule. In addition, migration of spindleshaped lens epithelial (fibre) cells into the retrolental space was noted in the early postoperative period (data not shown), which is consistent with previous studies. It thus may be that the weak adhesion of the hydrogel material to the capsule leads to these disadvantages.

In conclusion, contraction of the anterior capsule opening was greater with the hydrogel IOL than with the acrylic IOL. The area reduction with the hydrogel IOL was not so extensive as to disturb visual acuity. However, the reduced opening certainly limits the examination of the peripheral retina and also increases the difficulty of laser photocoagulation. Therefore, one should be particularly careful not to make a small capsulorhexis with the hydrogel IOL in patients with retinal morbidity. Furthermore, the open loop design hydrogel IOL shows good centration as long as implanted in the capsule. Therefore, surgeons should carefully place this lens into the capsular bag.

The authors have no proprietary interest in any of the materials described in this article.

1 Packerd RBS, Garner A, Arnott EJ. Poly-HEMA as a material for intraocular lens implantation: a preliminary report. Br f Ophthalmol 1981;65:585-7.

2 Barrett GD, Constable IJ, Stewart AD. Clinical results of hydrogel lens implantation. I Cataract Refract Surg 1986;12:623-31.

3 Amon M, Menapace R. Cellular invasion on hydrogel and poly(methyl methacrylate) implants: an in vivo study. $\mathcal{f}$ Cataract Refract Surg 1991;17:774-9.

4 Hollick EJ, Spalton DJ, Ursell PG. Surface cytologic features on intraocular lenses: can increased biocompatibilfeatures on intraocular lenses: can increased biocompatibil-

5 Meapace R, Yalon M. Exchange of IOGEL hydrogel one-piece foldable intraocular lens for bag-fixated J-loop poly(methyl methacrylate) intraocular lens. $\mathcal{f}$ Cataract Refract Surg 1993;19:425-30.

6 Menapace R, Skorpik C, Juchem M, et al. Evaluation of the first 60 cases of poly HEMA posterior chamber lenses implanted in the sulcus. F Cataract Refract Surg 1988;15: 264-71

7 Lowe KJ, Easty DL. A comparison of 141 polymacon (Iogel) and 140 poly(methyl methacrylate) intraocular lens implants. Br f Ophthalmol 1992;76:88-90.

8 Noble BA, Hayward JM, Huber C. Secondary evaluation of hydrogel lens implants. Eye 1990;4:450-5.

9 Menapace R, Papapanos P, Radax U, et al. Evaluation of 100 consecutive IOGEL 1003 foldable bag-style lenses implanted through a self-sealing tunnel incision. 7 Cataract Refract Surg 1994;20:432-9.

10 Hayashi K, Hayashi H, Nakao F, et al. Reduction in the area of the anterior capsule opening after polymethylmethacrylate, silicone, and soft acrylic intraocular lens implantation. Am f Ophthalmol 1997;123:441-7.

11 Ursell PG, Spalton DJ, Pande MV. Anterior capsule stability in eyes with intraocular lenses made of poly(methyl methacrylate), silicone, and Acrysof. 7 Cataract Refract Surg 1997;23:1532-8.

12 Milauskas AT. Posterior capsule opacification after silicone lens implantation and its management. $\mathcal{F}$ Cataract Refract Surg 1987;13:644-8.

13 Hayashi H, Hayashi K, Nakao F, et al. Quantitative comparison of posterior capsule opacification after polymethylmathacrylate, silicone, and soft acrylic intraocular lens implantation. Arch Ophthalmol 1998;116:1579-82.

14 Ursell PG, Spalton DJ, Pande MV, et al. Relationship between intraocular lens biomaterials and posterior capsule between intraocular lens biomaterials and posterior capsu

15 Hollick EJ, Spalton DJ, Ursell PG, et al. The effect of polymethylmethacrylate, silicone, and poleacrylic intraocuar lenses on posterior capsular opacification 3 years after cataract surgery. Ophthalmology 1999;106:49-55.

16 Menapace R. Posterior capsule opacification and capsulotomy rates with taco-style hydrogel intraocular lenses. $\mathcal{F}$ Cataract Refract Surg 1996;22:1318-30.

17 Hollick EJ, Spalton DJ, Ursell PG, et al. Posterior capsule opacification with hydrogel, polymethylmethacrylate, and silicone intraocular lenses: two-year results of a randomized silicone intraocular lenses: two-year results of a randomiz
pospective trial. Am f Ophthalmol 2000;129:577-84.

18 Hayashi K, Harada M, Hayashi $\mathrm{H}$, et al. Decentration and Hayashi $K$, Harada $M$, Hayashi $H$, et al. Decentration and
tilt of polymethyl methacrylate, silicone, and acrylic soft intraocular lenses. Ophthalmology 1997;104:793-8.

9 Levy JH, Piscano AM, Anello RD. Displacement of bag-placed hydrogel lenses into the vitreous following neodymium:YAG laser capsulotomy. F Cataract Refract Surg 1990;16:563-66.

20 Koch MU, Kalicharan D, van der Want JJL. Lens epithelial layer formation related to hydrogel foldable intraocular lenses. F Cataract Refract Surg 1999;25:1637-40.

21 Lenis K, Philipson B. Lens epithelial growth on the anterior surface of hydrogel IOLs: an in vivo study. Acta Ophthalmol Scand 1998:76:184-7.

22 Goarnisson S, Hennekes R. Medium term results of HEMA intraocular lenses (Hydroview ${ }^{\circledR}$ ). Bull Soc Belge Ophtalmol 1999;272:63-8.

23 Menapce R, Skorpik C, Wedrich A. Evaluation of 150 cases of poly HEMA posterior chamber lenses implanted in the bag using a small-incision technique. I Cataract Refract Surg 1990;16:567-77.

24 Menapace R, Amon M, Radax U. Evaluation of 200 consecutive Iogel 1103 capsular-bag lenses implanted through a small incision. 7 Cataract Refract Surg 1992;18: $252-64$.

25 Ishibashi T, Araki H, Sugai S, et al. Anterior capsule opacification in monkey eyes posterior chamber intraocular lenses. Arch Ophthalmol 1993;111:1685-90.

26 Kato K, Kurosaka D, Nagamoto T. Apoptotic cell death in rabbit lens after lens extraction. Invest Ophthalmol Vis Sci 1996;37:2322-30.

27 Marcantonio JM, Rakic JM, Vrensen GFJM, et al. Lens cell populations studies in human donor capsule bags with implanted intraocular lenses. Invest Ophthalmol Vis Sci 2000;41:1130-41. 